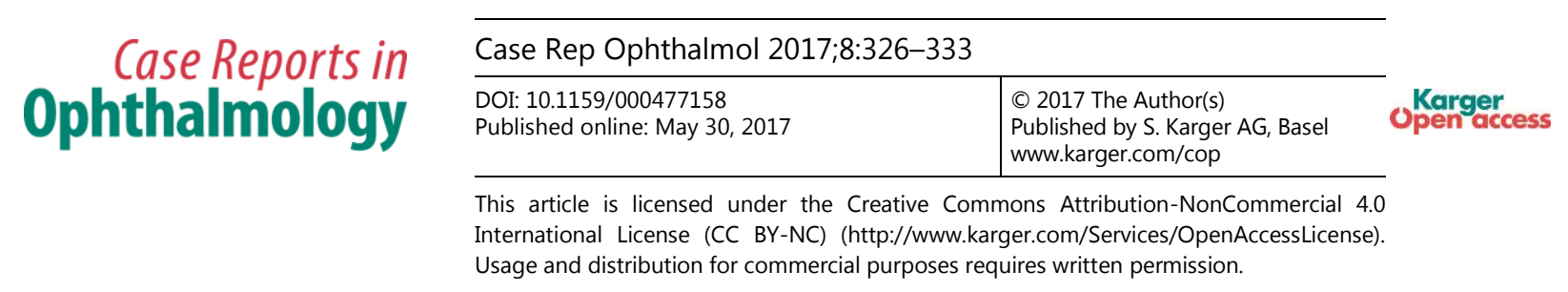

\title{
Shallow Anterior Chamber in a Severe Case of Unilateral Acute Central Serous Retinal Detachment
}

\author{
Ahmet Colakoglu Banu Cosar \\ Acibadem University School of Medicine, Istanbul, Turkey
}

\section{Keywords}

Central serous chorioretinopathy · Optical coherence tomography · Anterior chamber . Biometry . Anterior vitreous cells $\cdot$ Serous retinal detachment

\begin{abstract}
Background: Atypical serous chorioretinopathy can present with symptoms similar to those of other pachychoroid diseases; however, a proper differential diagnosis will prevent unnecessary intensive treatments. To this end, we report on the shallowness of anterior chamber and transitory anterior vitreous cells in a patient with atypical severe serous retinal detachment. Case Presentation: A 42-year-old woman presented with unilateral visual loss accompanied by mild, vague eye pain for 4 days. Spectral-domain OCT scans of the left eye showed macular and peripapillary serous detachment. Optical biometry showed the clinically significant shallow anterior chamber. Conclusions: Severe central serous chorioretinopathy can show up in an atypical fashion with macular, extramacular, juxtapapillary serous detachments, temporary existence of anterior vitreous cells, clinically detectable decreased anterior chamber depth, and mild eye pain of short duration.

(C) 2017 The Author(s) Published by S. Karger AG, Basel
\end{abstract}




\section{Background}

Central serous chorioretinopathy (CSC) is a disorder associated with leakage of fluid through the retinal pigment epithelium (RPE) into the subretinal space. The acute form generally resolves spontaneously with minimal sequelae. The hallmark of CSC is the presence of a serous detachment of the neurosensory retina in the posterior pole. These findings are easily detected with optical coherence tomography (OCT). Fluorescein angiography (FA) may show evidence of CSC episodes limited to the extramacular area that, because they are asymptomatic, typically go undetected. CSC can be regarded as a disorder of the posterior pole rather than a pure maculopathy [1]; indeed, CSC often extends beyond the macula [2]. Acute CSC is typically a self-limiting process [3]. The association between CSC and the type A personality is intriguing [4].

We report a case of acute unilateral CSC in which large macular and extramacular areas of serous retinal detachment were associated with a "clinically prominent" shallow anterior chamber (AC) and anterior vitreous cells. Here, we show the spectral OCT and biometric recordings of this case.

\section{Case Report}

A previously healthy 42-year-old woman presented to our clinic with blurring of vision and visual loss in the left eye (LE) for 4 days, associated with mild eye pain. There were no auditory, dermatological, or neurological symptoms. The patient's family, medical, and ocular histories were unremarkable, except for a type A behavioral pattern. She denied the use of drugs. She had been diagnosed at another clinic with presumed unilateral Vogt-KoyanagiHarada (VKH) disease. The patient was referred to our clinic for differential diagnosis, workup, and treatment before starting further therapy.

At the initial visit, her best-corrected visual acuity (BCVA) on presentation was 25/20 in the right eye and 20/40 in the LE. The patient's cycloplegic spherical equivalent error was $+0.50 \mathrm{dpt}$ on the right, and $-1.00 \mathrm{dpt}$ on the left. Intraocular pressure was normal, and no remarkable inflammation was observed in either the AC or posterior segment in both eyes, except for +1 cells in the anterior vitreous of the LE. A shallow left AC was readily apparent with slit-lamp microscopy, and was quantified with optical biometry.

The dilated funduscopic exam was unremarkable for the right eye, but the LE revealed large areas of retinal elevation consistent with subretinal serous fluid, as well mild optic disc hyperemia, and fuzzy margins of the optic disc. Serous retinal detachments were mainly located around the optic disc, extending up to the inferior and superior arcades beyond the posterior pole, short of equatorial region involving the papillomacular bundle, as well as the nasal retina and macula in the LE (Fig. 1a). Her right eye was fundoscopically normal.

We used spectral-domain OCT (3D OCT-1 Maestro, Topcon, Tokyo, Japan), which allows visualization of the retina, and ocular biometry measurements provided by an optical biometer (AL-Scan; Nidek Co, Ltd., Gamagori, Japan), which is based on partial coherence interferometry. Unfortunately, the OCT imaging did not include the enhanced depth imaging function needed to assess choroidal thickness. The patient refused FA, despite being informed 
about its potential use in the differential diagnosis. Neither fundus FA nor indocyanine green angiography were authorized by the patient.

OCT showed serous elevation of the sensory retina in the macular area involving the fovea, with papillomacular, juxtapapillary, peripapillary neurosensorial detachment (Fig. 1a). Anterior chamber depth (ACD) was shallower in the CSC eye than in the opposite eye by 0.62 $\mathrm{mm}$ at baseline. In addition, axial length was shorter in the CSC eye than in the opposite eye by $0.16 \mathrm{~mm}$ in the acute phase of the disease (Fig. 2a).

An initial diagnosis of atypical unilateral CSC was made based on the fundus appearance and spectral OCT findings, and the patient was followed closely and started on topical nepafenac ( $0.1 \%$ three times daily) administration to the LE for 2 months, until complete resolution of subretinal fluid and full recovery of visual acuity were achieved.

After taking a short holiday, the patient returned to the clinic with improved vision in the LE (20/32), as well as subjective and other objective visual improvements, including resolution of the ocular pain. Improvements were recorded as starting on day 12; refractive error was $+0.50 \mathrm{dpt}$ (SE) and $+0.25 \mathrm{dpt}$ (SE) in the right and LEs, respectively. Fundus examination demonstrated significant reduction in retinal elevations from the patient's previous exam. The OCT on day 12 demonstrated significant resorption of submacular fluid, as well as reduced elevation of the peripapillary retina (Fig. 1b). The difference between ACD of the right and left eyes decreased to $0.09 \mathrm{~mm}$ (Fig. 2b).

By day 37, BCVA in the LE improved to 20/25, with some mild subjective visual complaints. SE in the involved eye was determined to be $+0.00 \mathrm{dpt}$; in addition, anterior vitreous cells were absent. OCT displayed normal foveal contour and structure (Fig. 1c). The AC of the LE was just $0.07 \mathrm{~mm}$ deeper than the right eye (Fig. 2c).

On week 19, the patient's BCVA had improved to 20/20, without any residual subjective visual disturbances, and her ocular exam was unremarkable. Refractive error was $+0.50 \mathrm{dpt}$ in both eyes. The OCT showed complete resorption of the subretinal fluid and reestablishment of normal retinal anatomy.

After complete resolution of subretinal fluid, the ACD of the involved eye was only 0.06 $\mathrm{mm}$ shallower than the opposite eye. On the other hand, the left (involved eye) axial length was $0.07 \mathrm{~mm}$ longer than that in the opposite eye (Fig. 2d).

\section{Discussion}

Reports of spontaneous bullous retinal detachment in association with idiopathic CSC have been documented, albeit infrequently. This atypical presentation has been described as a severe, exaggerated form of idiopathic CSC, characterized by multiple leaking RPE detachments that are often obscured by fibrinous subretinal exudate. This uncommon variant sometimes may be incorrectly diagnosed as rhegmatogenous retinal detachment, VKH disease, uveal effusion syndrome, polypoidal choroidopathy, posterior scleritis, or multifocal chorioretinitis. In severe cases of idiopathic CSC in association with spontaneous bullous retinal detachment, fluid from multiple areas of leakage accumulates in the juxtapapillary subretinal space [5]. Thickened choroid may be present, representing the anatomical substrate supporting abnormal choroidal circulation in CSC [6]. How the enlarged choroid relates to CSC is unclear $[7,8]$.

Both VKH disease and CSC lead to serous retinal detachment. Although the pathogenesis of each disease is different, a thickened choroid can be seen in OCT imaging [8]. Bilateral 


\section{Case Reports in Ophthalmology}

involvement has been reported to be as high as $40 \%$ in CSC patients, although at the time of diagnosis, it has been reported as just 4\% [9].

VKH disease is defined as an inflammatory disease in the absence of ocular trauma or surgery, and without evidence of other ocular or systemic disease. VKH disease most commonly presents simultaneously in both eyes, although the second eye involvement may have delayed onset [10].

Strictly unilateral VKH disease is considered exceedingly rare. Also different from CSC, the treatment for VKH disease almost always requires high-dose systemic corticosteroids, and there are currently no reported cases of the disease spontaneously resolving [11]. Thus, in terms of differential diagnosis, unilaterality and complete resolution of retinal elevations without the need for corticosteroids are indicative of CSC, and not VKH disease.

The inflammatory changes of the anterior segment in VKH disease (i.e., ciliary edema and ciliochoroidal detachment) may exacerbate the shallow anterior chambers and narrow angles, and result in an acute increase in intraocular pressure in eyes with short axial length. VKH associated with shallow ACD may be misdiagnosed as acute primary angle closure [12].

Bilateral acute angle closure glaucoma can present as the initial symptom of VKH disease. Mildly increased intraocular pressure in association with visual disturbance in both eyes is an important sign of this diagnosis [13]. Notably, AC thinning may be a potential risk factor for induction of angle closure glaucoma in a patient with CSC and a critically narrow chamber angle; however, the collection of supporting evidence of this possibility will require future studies.

Oh et al. [14] investigated the biometric characteristics related to choroidal thickening and the effects of choroidal thickening on optical characteristics in CSC eyes. They demonstrated that ACD was significantly shallower in CSC eyes than in the opposite (unaffected) eyes. Hyperopic shift is a common finding that corresponds to central retinal elevation in CSC eyes [14]. However, our patient instead displayed a myopic shift that overwhelmed the hyperopic shift, which would be expected to occur as a result of the marked central retinal detachment.

Choroidal venous congestion in CSC eyes might be associated with ciliary body congestion and edema. Consequently, ciliary body edema would cause a forward shift of the lens, or an increase in lens thickness, and a shallow anterior chamber. Another possibility for the cause of shallow ACD in CSC eyes is accommodation by the eye with CSC, in which increased lens thickness and forward shifting of the anterior lens surface could cause the anterior chamber to become shallow [14].

Notably, however, at presentation, the spherical equivalent of refractive error in our case was consistently myopic after full cycloplegia was achieved. This finding eliminates accommodation as the sole reason for the reduced depth of the anterior chamber. However, it may be a contributing factor in increasing the forward movement of the lens; that is accommodation in combination with ciliary edema can lead to the myopic shift seen in our patient.

Ahmad et al. [15] reported a one-eyed patient with a history of blurred vision. Slit-lamp examination results of the anterior segment were normal. Ophthalmoscopy demonstrated $1+$ cells in the anterior vitreous and a serous retinal detachment involving the macula, and extending from the 2-o'clock to the 10-o'clock position with pigmentary and atrophic changes in the macula.

Considering the large serous detachment, widespread angiographic retinal vascular leakage, and vitreous cell, they suspected VKH disease and opted for a diagnostic trial of high-dose corticosteroid therapy. However, gradually increasing overall macular volume and 


\section{Case Reports in Ophthalmology}

subretinal fluid were revealed. They believed that this finding confirmed an underlying diagnosis of CSC. In retrospect, they inferred that the "vitritis" was secondary to the chronic retinal detachment with subsequent breakdown of the blood-retinal barrier, and was not a priori evidence of inflammatory disease.

In our case, we also observed 1+ cells in the anterior vitreous, which were undetectable as of the third visit. We accommodated our patient's reluctance to use systemic corticosteroids, and in combination with our preliminary diagnosis of severe CSC, chose not to try therapy-based testing, but instead to follow up the patient closely. Considering the transitory existence of these cells, we regarded them as being noninflammatory cells.

In contrast to the case of Ahmad et al. [15], our patient complained of a vague, mild eye pain, which had resolved by the second visit. We hypothesize that the slow progression of their case might have allowed enough time for intraocular tissues to adapt the volume in response to the large areas of increased choroidal thickness. The rate at which large areas of choroidal thickening develop may be more important than the extension of choroidal thickening as far as eyeball pain is concerned; a rapid increase in choroidal volume can both overwhelm the mechanisms of pain compensation in the eye, and lead to a shallow ACD.

The unique characteristics of our case included the "clinically" significant shallowness of the anterior chamber, the periocular pain, and the peripapillary severity of retinal elevation. Complete functional and structural recovery was achieved without using any therapy, except for instilling a topical nonsteroidal anti-inflammatory drug within 25 days. Such clinical yield is more in keeping with a diagnosis of CSC. Thus, the above characteristics differentiated our case from that of Ahmad et al. [15], while the strict unilaterality of our case and nonsteroidal resolution differentiated it from VKH disease.

We recognize that CSC should have been diagnosed after FA, in addition to OCT, for a complete differential diagnosis. However, we believe that this disadvantage was overcome by the retrospective diagnosis of CSC.

\section{Conclusions}

We identified and treated a unique case of unilateral severe CSC, with prominent anterior chamber shallowness and transitory anterior vitreous cells. OCT findings in the case were consistent with acute CSC. The exceptional rarity of these findings should be kept in mind in order to differentially diagnose suspicious cases from other pachychoroid conditions. Close follow-up with fundus examination and a detailed anterior segment, anterior vitreous examination at presentation could provide supportive evidence for the actual incidence of these signs in atypical CSC cases.

\section{Statement of Ethics}

The patient has consented to the submission of the case report to the journal.

\section{Disclosure Statement}

No funding was received for this research. The authors declare no conflict of interest. 
References

1 Nicholson B, Noble J, Forooghian F, Meyerle C: Central serous chorioretinopathy: update on pathophysiology and treatment. Surv Ophthalmol 2013;58:103-126.

2 Spaide RF, Hall L, Haas A, et al: Indocyanine green videoangiography of older patients with central serous chorioretinopathy. Retina 1996;16:203-213.

-3 Klein ML, Van Buskirk EM, Friedman E, Gragoudas E, Chandra S: Experience with nontreatment of central serous choroidopathy. Arch Ophthalmol 1974;91:247-250.

-4 Yannuzzi LA: Type A behavior and central serous chorioretinopathy. Trans Am Ophthalmol Soc 1986;84:799-845.

-5 Chen JC, Lee LR: Central serous chorioretinopathy and bullous retinal detachment: a rare association. Clin Exp Optom 2005;88:248-252.

-6 Moon H, Lee DY, Nam DH: Axial length in unilateral idiopathic central serous chorioretinopathy. Int J Ophthalmol 2016;9:717-720.

7 Lehmann M, Bousquet E, Beydoun T, Behar-Cohen F: Pachychoroid: an inherited condition? Retina 2015;35:10-16.

-8 Komuku Y, Iwahashi C, Yano S, Tanaka C, Nakagawa T, Gomi F: En face optical coherence tomography imaging of the choroid in a case with central serous chorioretinopathy during the course of VogtKoyanagi-Harada disease: a case report. Case Rep Ophthalmol 2015;6:488-494.

$>9$ Ross A, Adam H, Ross AH, Mohamed Q: Review and update of central serous chorioretinopathy. Curr Opin Ophthalmol 2011;22:166-173.

10 Read RW, Holland GN, Rao NA, Tabbara KF, Ohno S, Arellanes-Garcia L, Pivetti-Pezzi P, Tessler HH, Usui M: Revised diagnostic criteria for Vogt-Koyanagi-Harada disease: report of an international committee on nomenclature. Am J Ophthalmol 2001;131:647-652.

11 Li B, Bentham RJ, Gonder JR: A case of unilateral and spontaneously resolving posterior uveitis with overlapping features of Vogt-Koyanagi-Harada disease and acute posterior multifocal placoid pigment epitheliopathy. Springerplus 2016;5:1471.

-12 Takemoto D, Ijiri S, Shimizu M, Higashide T, Sugiyama K: Two cases of Vogt-Koyanagi-Harada disease presenting shallow anterior chamber. Nippon Ganka Gakkai Zasshi 2015;119:354-362.

-13 Yao J,, Chen Y, Shao T, Ling Z, Wang W, Qian S: Bilateral acute angle closure glaucoma as a presentation of Vogt-Koyanagi-Harada syndrome in four Chinese patients: a small case series. Ocul Immunol Inflamm 2013;21:286-291.

14 Oh J-H, Oh J, Togloom A, Kim S-W, Huh K: Biometric characteristics of eyes with central serous chorioretinopathy. Invest Ophthalmol Vis Sci 2014;55:1502-1508.

15 Ahmad S, Chen CJ, Campbell JP: Serous retinal detachment following enucleation. JAMA Ophthalmol 2015;133:713-714. 


\section{Case Reports in Ophthalmology}

Case Rep Ophthalmol 2017;8:326-333 DOI: $10.1159 / 000477158$

C 2017 The Author(s). Published by S. Karger AG, Basel www.karger.com/cop

Colakoglu and Cosar: Shallow Anterior Chamber in a Severe Case of Unilateral Acute Central Serous Retinal Detachment
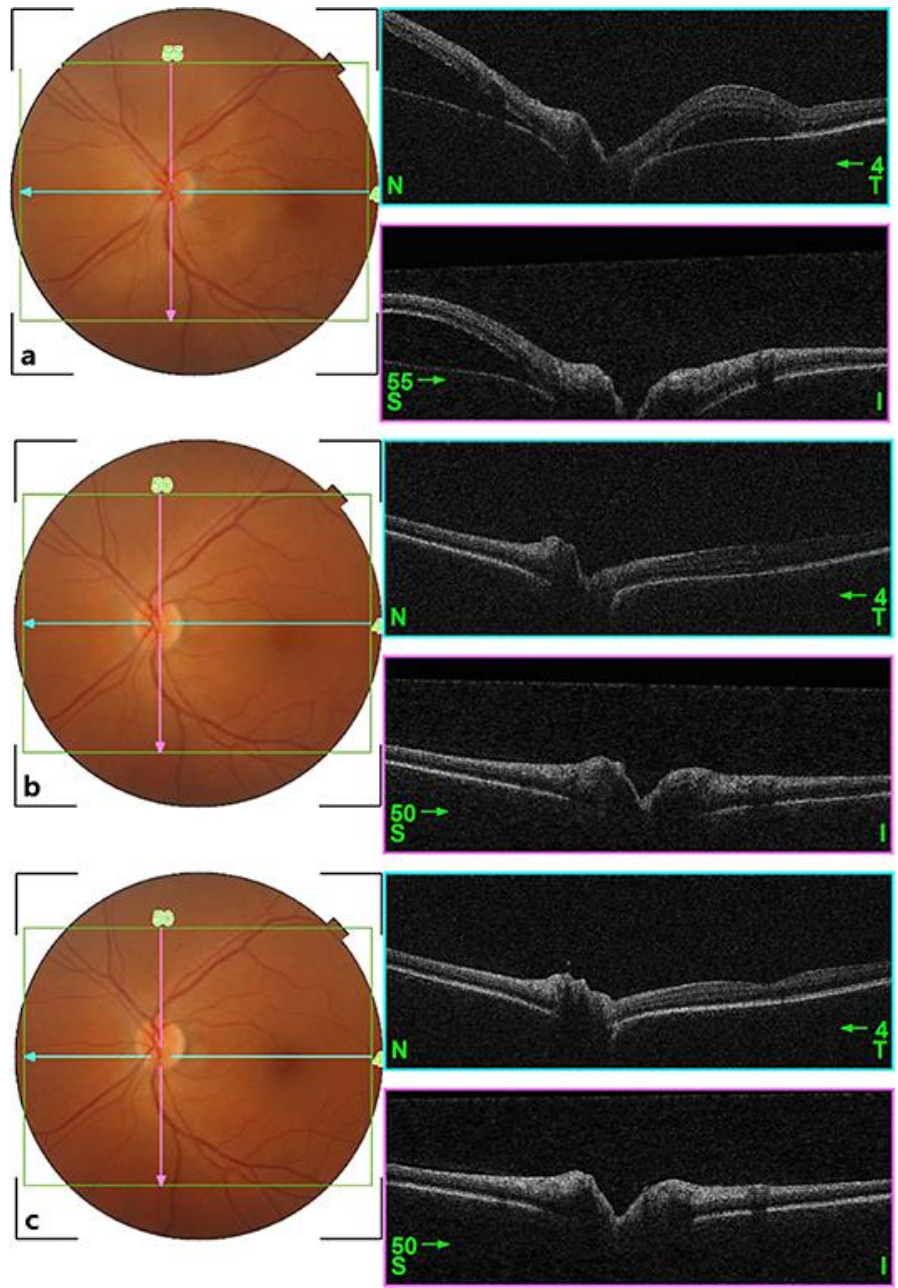

Fig. 1. OCT images during acute, resolving, and resolved phases. a Day 0 (initial visit): large macular and papillomacular neurosensory detachment with subretinal serous fluid. b Day 12: marked resolution of subretinal fluid. c Day 37: complete resorption of subfoveal space and reestablishment of retinal layers. 


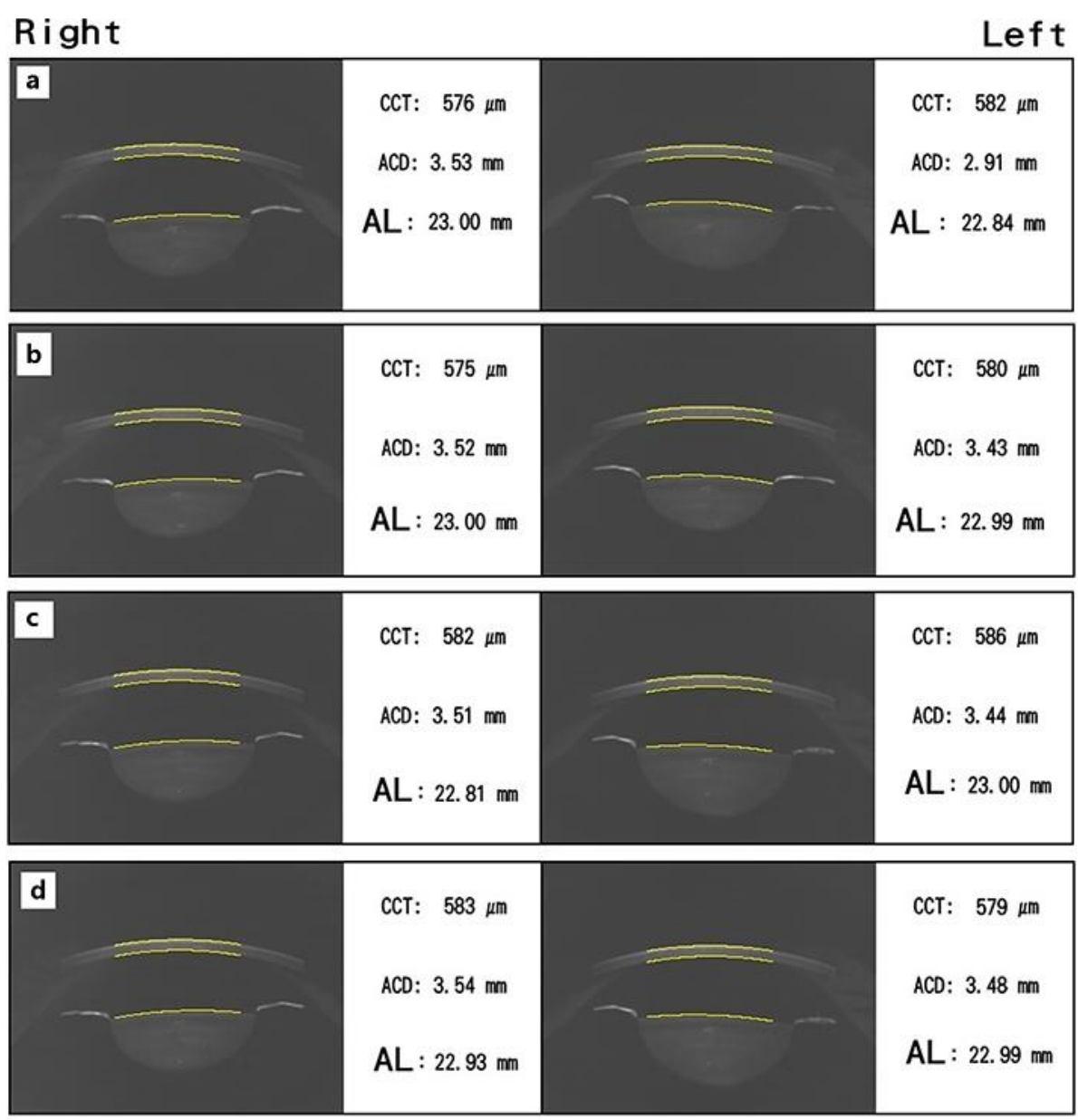

Fig. 2. a ACD was shallower in the CSC eye (left) by $0.62 \mathrm{~mm}$ at baseline. b, c Optical biometry on day 12 (b) and on day 37 (c). d In week 19, the ACD of the left eye was only $0.06 \mathrm{~mm}$ shallower than that of the right eye. 